\title{
The Processes of Public Safety Emergency Services System
}

\author{
Jianping Zhang ${ }^{+}$, Jianguo Chen, Chuanjie Yang, Hongyong Yuan \\ Institute of Public Safety Research, Department of Engineering Physics, Tsinghua University
}

\begin{abstract}
This article present the conventional process of public safety emergency services and then recommended in detail the business process of call-taking, incident assignment and dispatching in public safety emergency services system. A space-time analytical method for determining the repetitive alarm is provided. Alarm ways, call-taking information, incident assignment logic and disposal approaches also are discussed.
\end{abstract}

Keywords: Emergency services, call-taking, dispatching

\section{Introduction}

Natural disasters, traffic accidents, environment pollution, cyber incidents and terrorism can all cause emergencies in a city [1,2]. They may quickly escalate in scope and severity, cross jurisdictional lines and take on international dimensions. Currently, governments and the public safety emergency service organizations are trying to improve service and public confidence at a time when the threat of various incidents remain high and the public scrutiny is at its most intense, which is an unenviable challenge.

The public safety emergency services system represents a dynamic configuration of an unlimited number of entities with the goal of ensuring secure conditions for all entities operating on the specific administrative territory using resources possessed within the framework of formal rules and informal relationships, typified by non-repeatability and volatility as well as continuous adjustment to current circumstances and needs occurring [3]. For the fast and efficient response once a public safety incident occurs, a uniform public safety emergency services system is needed $[4,5]$. Some successful cases have been applied in some countries and regions, such as the 911 center of America, European emergency number 112, the emergency service 999 of the United Kingdom, the emergency service 000 of Australia and so on. And some novel systems are being developed [6-8].

The rest of this paper is organized as follows. Section II describes the process of public safety emergency services. Section III discusses the key information in call-taking process. Section IV analyse the incident assignment logic. Section V proposes the dispatching process in public safety emergency services. Section VI provides the concluding remarks.

\section{Process of Public Safety Emergency Services}

In order to discuss the application of business processes of public safety emergency services, we should know the process of public safety emergency services first. Take a fire alarm for instance. When a fire occurs in a building, citizens on-site may alert to the public safety answering point by taking a call, send a message or some other approaches, the monitoring equipment in or near the building will also alarm to the public safety answering point. Call-taker in the public safety answering point would receive the incident and record the related information, then assign the incident to the corresponding dispatchers, the fire dispatcher and the medical dispatcher if casualties reported in this incident. The dispatchers will assemble different response

\footnotetext{
+ Corresponding author. Tel.: 86+15101544894;
}

E-mail address: jpzhang@live.com. 
force, the firefighters, fire engines, medical staffs and ambulances in this case. Then the dispatched response force will rush to the scene and deal with the accident.

\section{Process of Public Safety Emergency Services System}

\subsection{Process of Call-Taking in Public Safety Emergency Services System}

When an incident occurs, there may be various alarm ways, as shown in figure 1 . Take a fire accident as an example, citizens may call the public safety emergency center by cell phone or landline, or send a short message to the public safety emergency center. The affected merchant may give an alarm by an intelligent merchant terminal, while the community residents may give an alarm by the intelligent community terminal. If there is a video alarm pole, people could also give an alarm by the video alarm pole. It is also possible that a police is patrolling nearby, he may contact with the public safety emergency center though the carry-on police terminal. There may be a police station, witnesses may report directly to the police station, if the officers on-duty cannot deal with the incident, they may summit is to the upper public safety emergency center. Meanwhile, the incident may also occur in some other places, such as a robbery occurs on a bus, or a wanted vehicle is found at a traffic bayonet, the corresponding monitoring system may send an alarm to the public safety emergency center.

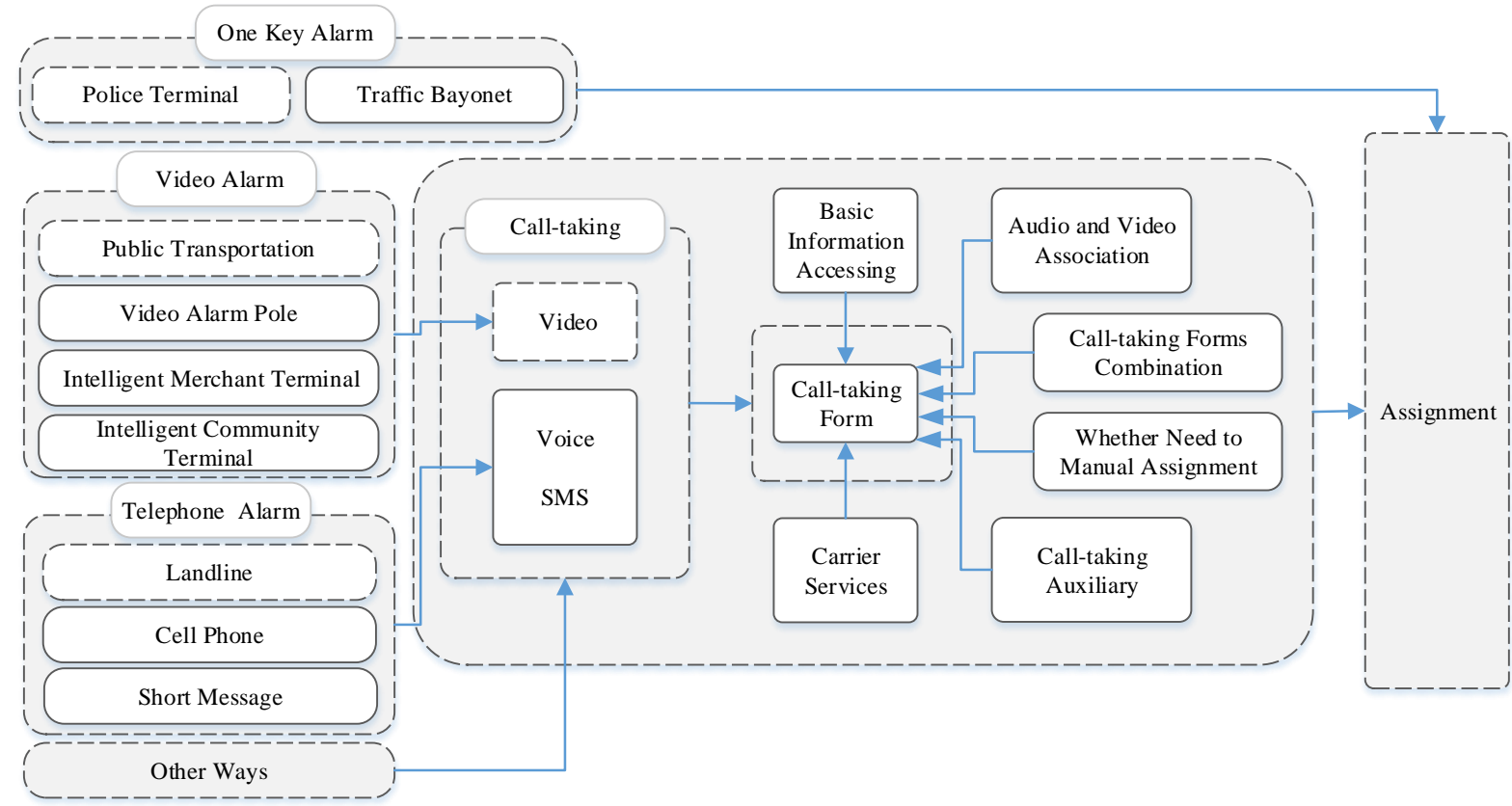

Fig. 1: Process of call-taking

When the public safety emergency center receives an alarm, the call-taker would fill in the call-taking form, in which including the alarm person's information, the incident information and relevant description. Take a telephone alarm for example, the alarm person's information may involve the phone number, the alarm address, the caller's name, sex and age, etc. The incident information need to define the incident type, the occurrence time, address, and urgency level. The relevant description need to describe other details such as casualties, sweep range and so on.

The basic information such as the phone number and the alarm address could be accessed from the carrier services and filled in the form automatically if it is possible. The audio and video files of the alarm would also be associated to the call-taking form. If there are more than one alarms for an incident, the calltaker will combine them into one. The basis for call-taking forms combination are distance, time and the key words of the incident. A space-time analytical method is proposed here, as shown in figure 2. 


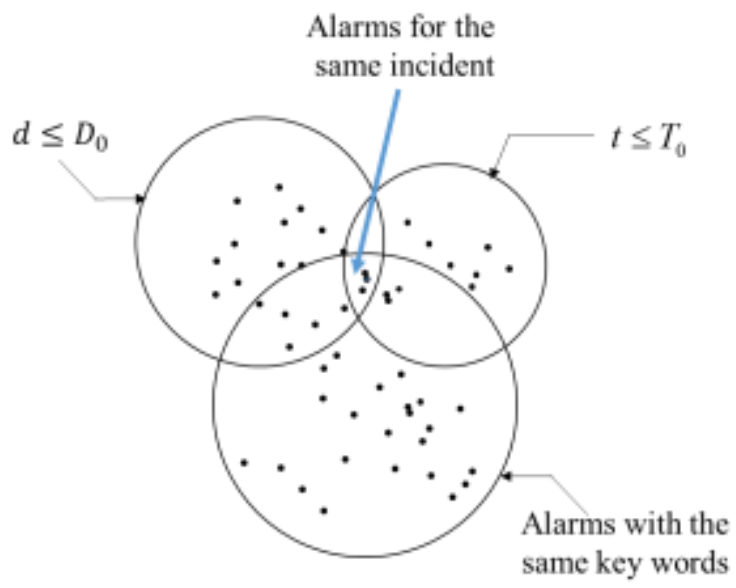

Fig. 2: Space-time analytical method for call-taking forms combination

Suppose $A_{\mathrm{D}}$ is the set of alarms with a distance di to the current alarms' site less than a certain value, i.e.

$$
A_{\mathrm{D}}=\left\{a_{d_{i}}\right\}, d_{i} \leq D_{0}
$$

And $A_{\mathrm{T}}$ is the set of alarms within a time $T_{0}$ of the current alarms' time, i.e.

$$
A_{\mathrm{T}}=\left\{a_{\mathrm{T}_{j}}\right\}, t \leq T_{0}
$$

Similarly, $A_{\mathrm{skw}}$ is the set of alarms have the same key words with that of the current alarms.

$$
A_{\mathrm{skw}}=\left\{a_{\mathrm{skw}}\right\} \text {, the same key wards }
$$

Here $i, j, \mathrm{k}$ is the index of alarms.

Then alarms for the same incident $A_{\text {same }}$ could be described as follow

$$
A_{\text {same }}=A_{\mathrm{D}} \cap A_{\mathrm{T}} \cap A_{\text {skw }}=\left\{a_{d_{i}}\right\} \cap\left\{a_{\mathrm{T}_{j}}\right\} \cap\left\{a_{\text {skw }_{k}}\right\} \quad d_{i} \leq D_{0}, t \leq T_{0} \text {, the same key wards }
$$

In addition, the call-taker will judge whether the incident need to manually assign to the specific dispatcher. If not, the incident will be assign to an appropriate dispatcher according to certain logic as discussed in the next section. A professional and efficient call-taking auxiliary could help the call-taker quickly complete the call-taking form. It can be possible if the system reminds the call-taker what and how to talk to the caller and obtain valid information.

\subsection{Process of Incident Assignment in Public Safety Emergency Services System}

The business process of incident assignment is shown in figure 3. When a call-taking form was formed, the call-taker will firstly judge whether the incident need to manually assign to a specific dispatcher. If not, the system will assign it to a suitable dispatcher according to several assignment logics. First, every incident will be classified to fire, police, medical, traffic and some other types, incident of each type will be assigned to the corresponding dispatchers.

There may be three kinds of common assignment logic. The simplest is to randomly assign an incident to a dispatcher of its type. And some supplementary rules could be employed in this process. For example, the task cannot be assigned to the dispatcher whom was last assigned to. Or assign an incident to a free dispatcher rather than to a busy dispatcher. If all the dispatchers are busy, the system need to calculate the workload of all the dispatchers and assign the incident to whom with minimum workload. The amount and the urgency level of undisposed incident should be considered when calculate the workload of a dispatcher.

Suppose $N=\left\{n_{1}, n_{2}, \cdots n_{p}\right\}$ is the set of the undisposed incident number, $i$ is the index of dispatcher, $\mathrm{p}$ is the number of the dispatchers, $n_{i}$ is the undisposed incident number of dispatcher $i . u_{i j}$ is the urgency level of undisposed incident, $j$ is the index of undisposed incident of dispatcher $i$. Then the workload L of dispatcher $i$ can be described as follow

$$
L_{i}=\sum_{j=1}^{n_{i}} u_{i j} \quad(i=1,2, \cdots p)
$$


The target dispatcher whom should be assign to must be with the minimum workload, i.e.

$$
\text { Target }=\min \left(L_{i}\right)=\min \left(\sum_{j=1}^{n_{i}} u_{i j}\right) \quad(i=1,2, \cdots p)
$$

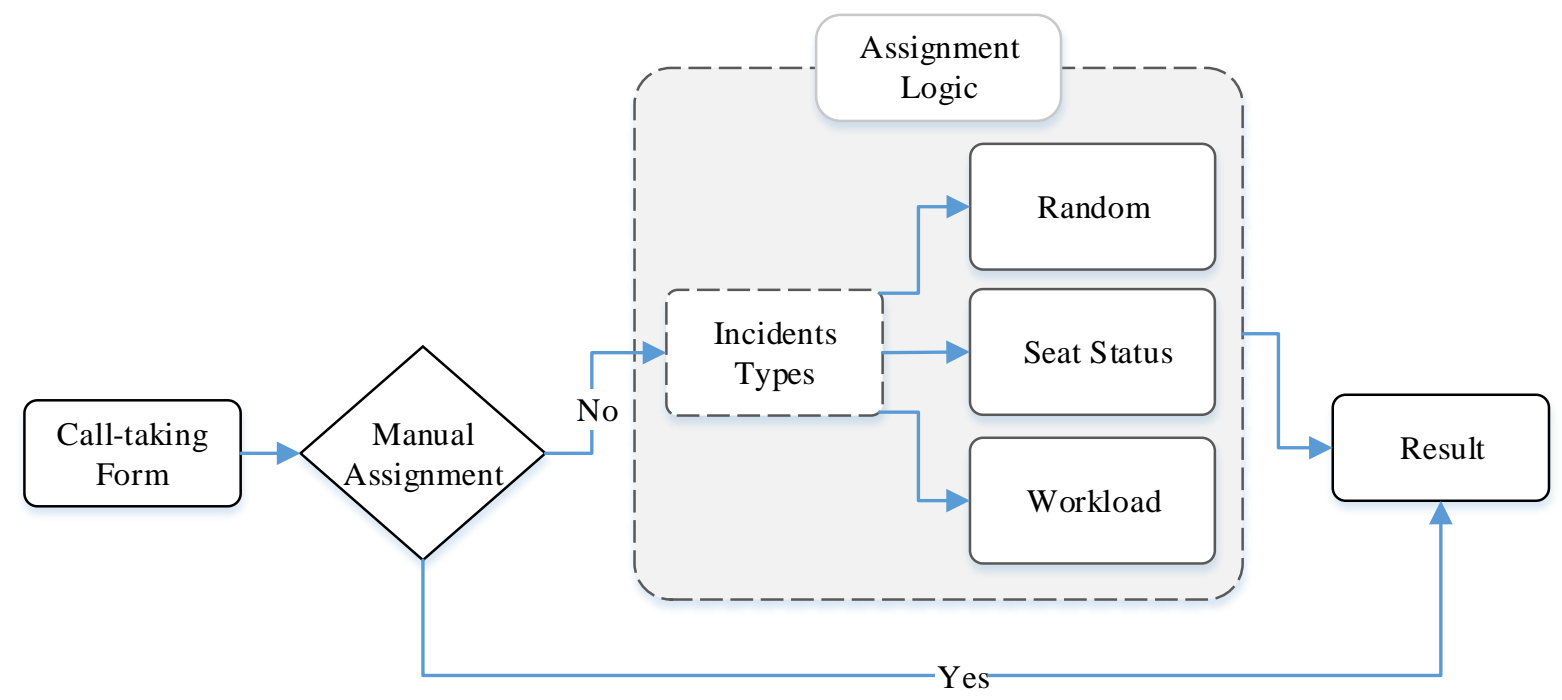

Fig 3: Process of incident assignment

\subsection{Process of Dispatching in Public Safety Emergency Services System}

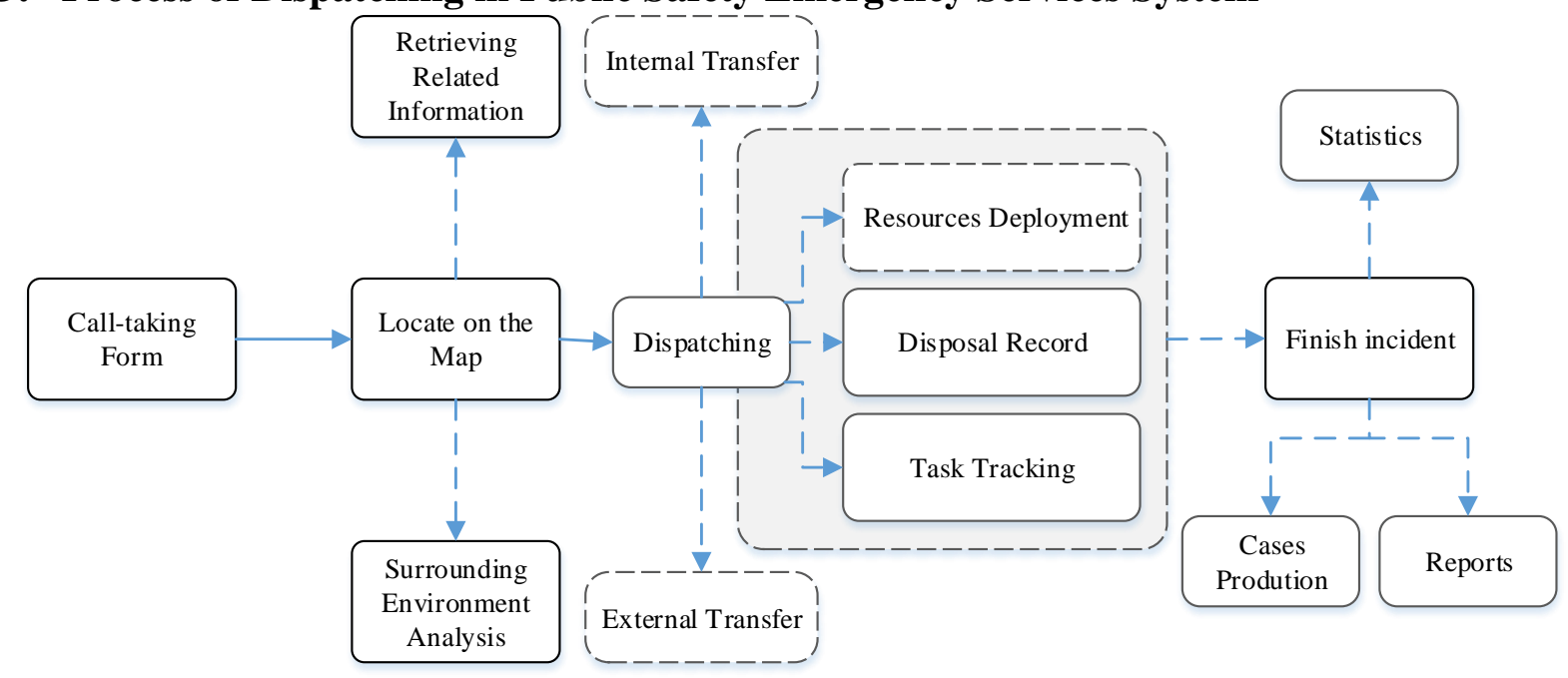

Fig 4. Process of Dispatching

When a dispatcher is assigned to an incident, he will begin to dispose it according to the process shown in figure 4. Usually, the system can automatically locate the incident on the map in the geographic information system (GIS). The dispatcher may firstly check the location. Then he could be retrieving information related to the incident. The related information may be the recordings of call-taking, the accessed video and pictures, or some other else information for helping understand the incident. If the incident is out of his duty, he may apply internal transfer or external transfer depending on the situation. Otherwise, he need to analyse the surrounding environment. And the surrounding environment analysis may provide knowledge such as the key protection units and the surrounding available resources.

After the dispatcher learn about information of the incident, he will begin to deploy resources to dispose the incident. Take a fire alarm for example, if the fire accident produced some casualties, the dispatcher need to apply a medical dispatcher for union. Then they need to deploy fire resources like firemen, fire engines, fire extinguisher and so on. Meanwhile, medical resources, such as medical staffs, ambulance, and other necessary medical equipment, should be deployed at the same time. For an efficient system, it will provide effective real-time communication channels. With these channels, deployed recourses could timely feedback 
the situation in scene. Meanwhile, the dispatchers could track the task at any time and adjust the disposal when necessary. Without doubt all the deployment recordings, tracking and feedback will be recorded in the system automatically.

After finished the incident, the system would create a report about the incident. If the incident and its disposal is representative, a relevant cases report will be produced. Statistical analysis of incident types, regional distribution, and time relationships and so on could be done.

\section{Conclusion}

The conventional process of public safety emergency services is described in this paper. And the business process of call-taking, incident assignment and dispatching in public safety emergency services system is discussed. A space-time analytical method for determining the repetitive alarm is provided. The development of technology diversifies the alarm ways and makes the dispatching quicker. Reasonable and scientific logic of incident assignment makes emergence respond more efficient.

\section{Acknowledgements}

This work was supported by National Key Technology Research and Development Program of the Ministry of Science and Technology of China (Grant No.: 2015BAK10B04).

\section{References}

[1] J. Santos-reyes, T. Gouzeva, G. Santos-reyes. Earthquake risk perception and Mexico City's public safety. Procedia Engineering. 2014, 84: 662-671.

[2] JH. Jin, ST. Zhao, B. Hu. Defining the Safety Integrity Level of Public Safety Monitoring System Based on the Optimized Three-dimension Risk Matrix, Procedia Engineering, 2012, 43: 119-124.

[3] S. Malyjurek, K. Kozuch, B. System zarzadzania bezpieczenstwem publicznym w ujeciu teorii zlozonosci, Opracowanie modelowe. Bezpieczenstwo i Technika Pozarnicza, 2015, 37: 33-43.

[4] K.S. Malyjureka. Determinants and attributes of leadership in the public safety management system. Procedia Economics and Finance. 2016, 39: 115-121.

[5] B. Kożuch, K.S. Małyjurek. New Requirements for Managers of Public Safety Systems. Procedia - Social and Behavioral Sciences. 2014, 149: 472-478.

[6] G. Bartoli, R. Fantacci, F. Gei, D. Marabissi and L. Micciullo. A novel emergency management platform for smart public safety. International Journal of Communication Systems. 2013, 28: 928-943.

[7] P. Francesco, M. Ficco, S. Pardi and A. Castiglione. A cloud-based architecture for emergency management and first responders localization in smart city environments. Computers and Electrical Engineering. 2016, 56: 810-830.

[8] J. Hayes. Taking responsibility for public safety: How engineers seek to minimise disaster incubation in design of hazardous facilities. Safety Science, 2015, 77:48-56. 
